\title{
1С: ПРЕДПРИЯТИЕ КАК ПРЕДМЕТНО-ОРИЕНТИРОВАННАЯ СРЕДА РАЗРАБОТКИ ИНФОРМАЦИОННЫХ СИСТЕМ
}

\section{C: ENTERPRISE AS A SUBJECT-ORIENTED ENVIRONMENT FOR THE DEVELOPMENT OF INFORMATION SYSTEMS}

\section{E. Nikonova M. Zabudskaya}

Summary. The article discusses the evolution of approaches to software development, ranging from the presentation of algorithms in the form of block diagrams and ending with a model-driven architecture. The types of models used in the process of modeling the subject area are highlighted. The authors analyze the capabilities of the 1C: Enterprise system for designing an information system, note its inherent advantages and limitations as a domain-specific development environment, determine the composition of the platform-dependent model created in the process of configuring the system, the types of objects used for modeling the domain.

Keywords: subject area, modeling, design automation, information system, technological platform. 1C: Enterprise.

\author{
Никонова Елена Захаровна \\ К.п.н., Нижневартовский государственный \\ университет \\ niko_len@mail.ru \\ Забудская Мария Петровна \\ Нижневартовский государственный универси- \\ mem \\ swimdeep23@gmail.com
}

Аннотация. В статье рассматривается эволюция подходов к разработке программного обеспечения, начиная от представления алгоритмов в виде блок-схем и заканчивая архитектурой, управляемой моделью. Выделяются типы моделей, используемых в процессе моделирования предметной области. Авторы анализируют возможности системы «1С: Предприятие» по проектированию информационной системы, отмечают свойственные ей как предметно-ориентированной среде разработки преимущества и ограничения, определяют состав платформенно-зависимой модели, создаваемой в процессе конфигурирования системы, типы объектов, используемых для моделирования предметной области.

Ключевые слова: предметная область, моделирование, автоматизация проектирования, информационная система, технологическая платформа. 1С: Предприятие. сновой современных технологий разработки информационных систем являются модели, представляющие внутреннюю структуру предметной области в виде графической и текстовой информации. Такой подход к разработке программного обеспечения далеко не нов. Еще в 40-х годах прошлого столетия были предприняты попытки формализовать и визуализировать процесс разработки программного обеспечения с помощью блок-схем, предложенных J. von Neumann. [5]

На смену блок-схемам пришел созданный в конце 90-х годов стандарт визуального моделирования ПО под названием UML (Unified Modeling Language) и основанные на нем методы разработки ПО RUP (Rational Unified Process) [6] и USDP (Unified Software Development Process).
Обязательным условием создания качественного программного продукта является наличие такой модели, которая достаточно точно отражает особенности предметной области и предоставляет разработчикам всю необходимую им информацию. Поэтому следующим этапом можно считать появление предметно-ориентированного моделирования (Domain-Specific Modeling, DSM), подразумевающего разработку модели предметной области в терминах самой области.

Результатом эволюции подходов к разработке ПО стала архитектура MDA (Model Driven Architectur), архитектура, управляемая моделью, представляющая данный процесс как последовательность этапов построения модели предметной области и последующего программирования на ее основе. [2] К преимуще- 


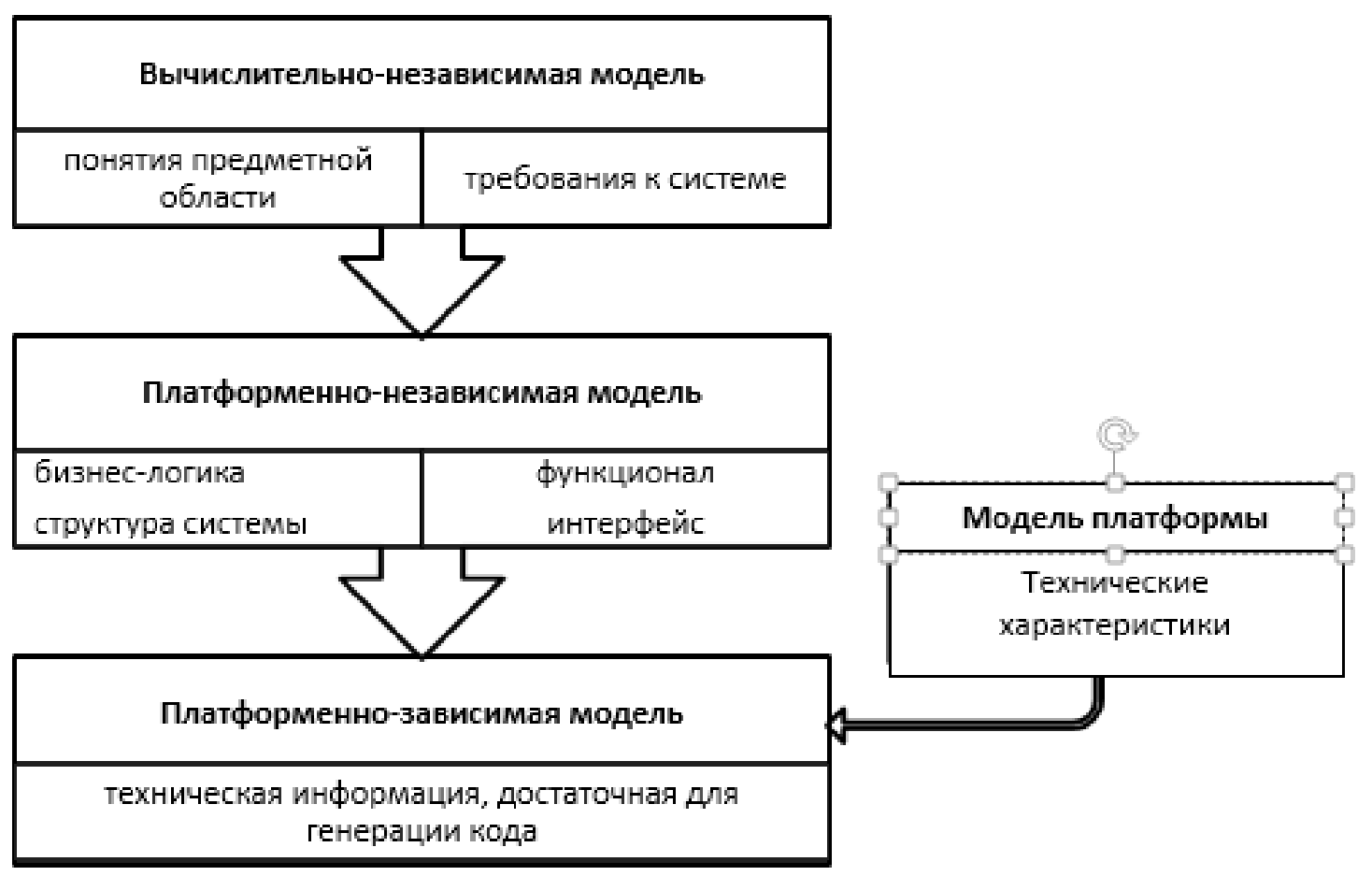

Рис. 1. Этапы преобразования моделей

ствам такого способа следует отнести, прежде всего, возможность создания по одной модели приложений для различных программных платформ, а также некоторую степень автоматизации программирования отдельных стандартных частей приложений, таких как создание базы данных или пользовательского интерфейса.

На этапе программирования модель предметной области постепенно уточняется, дополняется новыми деталями, т.е. процесс разработки представляет собой переход от одного вида модели к ее следующей ступени.

В архитектуре MDA модели могут описывать проектируемую систему на различных уровнях:

- уровень бизнес-логики, представляющей функциональность приложения;

- уровень данных, описывающих структуру данных и методы доступа к ним;

- уровень пользовательского интерфейса, включающий формы и элементы управления приложением.

В зависимости от степени реализации модели могут быть следующих типов:

- вычислительно-независимая модель (ВНМ) (Computation Independent Model - CIM), включающая только общие требования к системе в понятиях предметной области;
- платформенно-независимая модель (ПНМ) (Platform Independent Model - PIM), описывающая систему на каком-либо формализованном языке, например, UML;

- платформенно-зависимая модель (ПЗМ) (Platform Specific Model - PSM), описывающая структуру и функционал проектируемой системы с учетом особенностей той программной платформы, на которой будет реализована система (модели платформы).

Таким образом, в процессе разработки исходная модель предметной области проходит следующие этапы преобразования (см. рис. 1):

Согласно архитектуре MDA на этапе разработки платформенно-зависимой модели разработчики работают уже в конкретной среде разработки. Здесь следует отметить тенденцию перехода от набора специализированных инструментальных средств разработки (компиляторов, профилировщиков и т.п.) к системам, включающим максимально полный комплекс инструментов разработчика.

Одним из примеров подобных систем является предметно-ориентированная среда разработки 1С: Предприятие, обеспечивающая разработчика необходимым инструментарием для разработки и администрирования прикладного решения, автоматизирую- 
щего бизнес-процессы предприятия. Преимуществом 1C: Предприятия является не столько функциональность отдельных механизмов платформы (универсальные и специализированные средства разработки могут обладать гораздо большими возможностями), сколько их достаточное разнообразие и тесная интеграция. [1]

В частности, В состав технологической платформы входят такие инструменты:

- средства для визуального проектирования структур данных, запросов, интерфейса;

- редактор и отладчик программного кода,

- средства описания отчетов,

- инструменты администрирования и ролевой настройки прав пользователей;

- средства для работы по обновлению приложений, их сравнению и объединению,

- инструменты создания мобильных и Web-приложений,

- средства поддержки коллективной разработки,

- средства создания распределенных информационных систем и т.д.

Отметим следующие особенности среды 1С: Предприятие:

1. Наличие общей технологической модели работы приложения, включающей его трехуровневую архитектуру, методы взаимодействия компонентов системы, возможности аутентификации пользователей и т.п., которая предоставляется разработчикам в виде готовых архитектурно-технологических решений.

2. Подход к разработке приложения в виде описания структуры метаданных (metadata-driven), предполагающий в случае необходимости описание в определенных узлах с помощью программного кода бизнес-логики приложения или переопределения стандартного поведения объектов системы.

3. Конструирование прикладной модели приложения из стандартных прототипов -объектов конфигурации, каждый из которых обладает определённой функциональностью и служит для моделирования тех сущностей предметной области, которые схожи по своим свойствам и значению в бизнес-логике. Так как все типы объектов четко определены, разработчикам не приходится тратить усилия на определение их свойств и методов, все выполняется платформой автоматичеСКи.

4. Использование общей технологической модели и прикладной модели, с одной стороны, логически связанных, а с другой - достаточно независимых в технической реализации, что позволяет модифицировать технологическую платформу без изменения прикладной модели и приложения.

5. Возможность использования актуальных технологий (полнотекстовый поиск, Web-сервисы, XDTO, кластер серверов, управление блокировками данных и т.п.) в виде готовых механизмов, интегрированных в общую функциональность системы и не требующих длительного освоения.

6. Наличие системы обмена данными на основе $\mathrm{XML}$, что позволяет реализовывать территориально-распределенные информационные системы или осуществлять интеграцию как с другими приложениями 1С: Предприятия, так и со сторонними приложениями.

7. Возможность организации совместной работы пользователей приложения с помощью механизма бизнес-процессов.

8. Реализация data-mining для выполнения задач бизнес-анализа.

9. Возможность отображения географических карт для визуализации аналитических сведений или транспортной логистики по регионам.

Таким образом, проектирование информационной системы на основе технологической модели, метаданных и прикладной модели позволяет переложить значительную часть технических задач по реализации на платформу, а также минимизировать усилия разработчиков по осуществлению взаимодействия компонентов системы между собой, например, при построении отчетов на основе запросов.

Объективность требует отметить и ограничения предметно-ориентированной среды разработки, которые заключаются в строго определенном наборе технологических решений и возможностях их настройки, примером чего служит отсутствие прямого доступа к базе данных.

Рассмотрим более подробно состав платформенно-зависимой модели в 1С: Предприятие.

Основой для проектирования всех прикладных решений (конфигураций) является технологическая платформа, представляющая собой набор исполняемого файла, динамических библиотек и вспомогательных файлов. Именно благодаря наличию технологической платформы реализуются все рассмотренные выше достоинства 1С: Предприятия как предметно-ориентированной среды разработки. Кроме этого, использование общей методологии позволяет подстраивать стандартные решения под индивидуальные особенности конкретных предметных областей. 


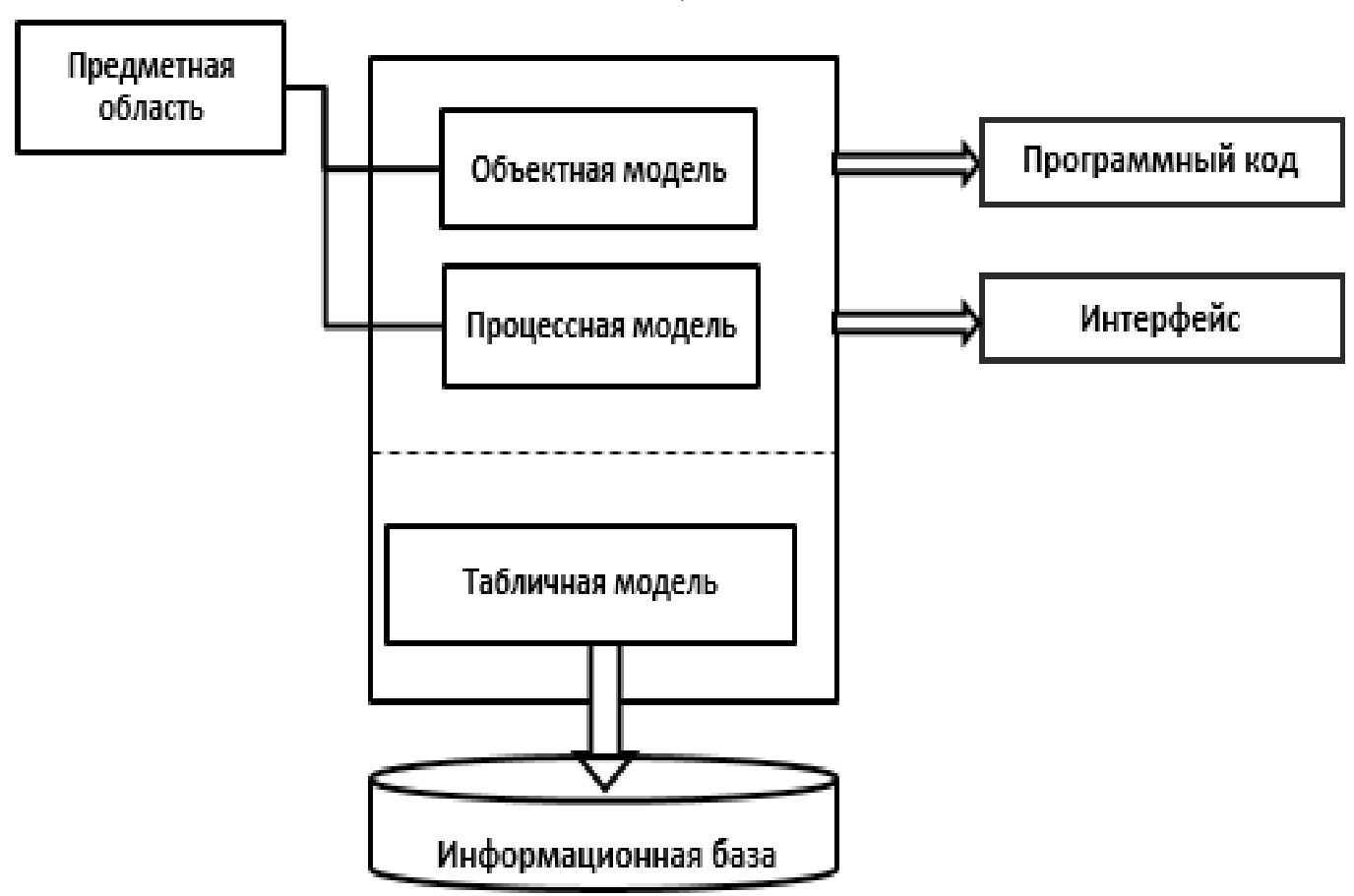

Рис. 2. Платформенно-зависимая модель в 1С: Предприятие

Моделью предметной области в системе 1С: Предприятие является конфигурация, включающая в себя структуру метаданных и программный код. Разработка конфигурации происходит в конфигураторе - среде разработки 1С: Предприятие с помощью специального инструмента - окна дерева конфигурации, включающего в себя объекты конфигурации.

По функциональности объекты конфигурации делятся на следующие виды:

- основные объекты (справочники, документы, отчеты и т.п.), с помощью которых моделируют отдельные сущности предметной области;

- подчиненные объекты, уточняющие структуру и поведение основных объектов (реквизиты, табличные части, формы, макеты);

- общие объекты, описывающие свойства конфигурации в целом.

Состав основных объектов обеспечивает наиболее эффективное моделирование задач административно-хозяйственного учета и документооборота, что и позволяет считать систему 1С: Предприятие предметно-ориентированной средой разработки.
Совокупность основных объектов представляет собой объектную модель предметной области, отражающую набор сущностей предметной области.

Логика взаимодействия пользователей представляется в виде процессной модели, содержащей специальные виды основных объектов - бизнес-процессов и задач.

Добавление каждого основного объекта (и некоторых подчиненных, таких как табличная часть) сопровождается автоматическим созданием таблицы данных в информационной базе конфигурации. Совокупность всех таких таблиц образуют табличную модель, к которой разработчик может обращаться при помощи механизма запросов.

При создании и настройке свойств объектов технологическая платформа автоматически формирует программные объекты, добавляет элементы управления и т.п.

Таким образом, платформенно-зависимая модель в системе 1С: Предприятие может быть представлена в виде следующей схемы (рис. 2):

\section{ЛИТЕРАТУРА}

1. Габец А.П., Гончаров Д.И., Радченко М.Г. Профессиональная разработка в системе 1С: Предприятие 8. — М:»1С-Паблишинг», 2006. — 808 с.

2. Остервальдер, А. Построение бизнес-моделей. Настольная книга стратега и новатора / А. Остервальдер, И. Пинье.- Альпина Паблишер, $2012 .-288$ с. 
3. Кознов, Д.В. Визуальное моделирование в менеджменте информационных технологий: введение / Д.В. Кознов // Математические модели и информационные технологии. - 2004.- № 2.- С. 75-86.

4. Автоматизированные информационные технологии в экономике. Под ред. Г.А. Титаренко, М., Компьютер, 1998. — c. 3-4, 98-100

5. Neumann, J. Planning and coding problems for an electronic computing instrument, part II, vol I. Rep. / H.H. Goldstein, J. Neumann // Prepared for the U.S. Army Ordinance Dept., Reprinted in von Neumann, J. Collected Works, Vol. V, A.H. Taub, Ed., Mc-Millan, New York. - 1947. - P. 80-151.

6. Kroll, P. The Rational Unified Process Made Easy: A Practitioner's Guide to the RUP: A Practitioner's Guide to the RUP / P. Kroll, P. Kruchten. —Addison-Wesley Professional, 2003. - $464 \mathrm{p}$.

( ) Никонова Елена Захаровна ( niko_len@mail.ru ), Нижневартовский государственный университет ( swimdeep23@gmail.com )

Журнал «Современная наука: актуальные проблемы теории и практики»

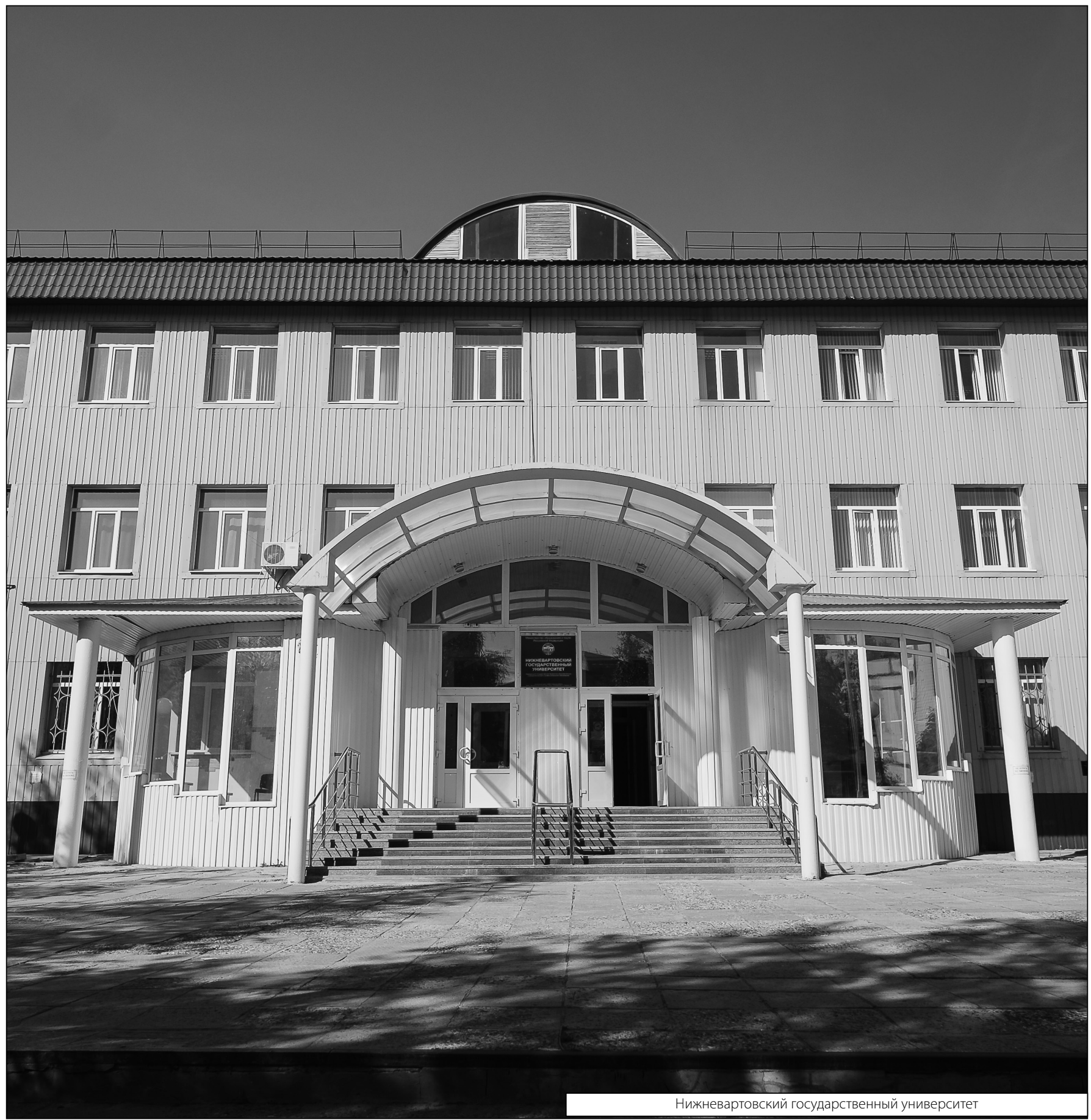

